\title{
El automonitoreo de la glucemia no mejoraría el control en pacientes con diabetes tipo 2 sin requerimiento de insulina
}

\author{
Glycemic self monitoring wouldn't improve control in patients with non-insulin treated diabetes.
}

Farmer A y col. BMJ 2007; 335: 132-6

\section{Objetivo}

Determinar si el automonitoreo de la glucemia en diabéticos tipo 2 no insulino requerientes, con o sin instrucciones para modificar su tratamiento, es más efectivo que los cuidados habituales en mejorar sus controles glucémicos.

\section{Diseño}

Estudio aleatorizado y abierto en tres grupos paralelos.

\section{Lugar}

48 médicos generales en Oxfordshire y South Yorkshire, Inglaterra.

\section{Participantes}

453 pacientes diabéticos tipo 2 con tratamiento no insulínico por un tiempo medio de tres años (edad promedio: 65,7 años) y un nivel de de hemoglobina glicosilada ( $\mathrm{HbA1c}$ ) promedio de $7,5 \%$.

\section{Intervención}

Fueron asignados a tres ramas de tratamiento: 1) cuidados habituales con medición de HbA1c cada tres meses ( $n=152)$; 2 ) cuidados habituales más autocontrol de baja intensidad con automonitoreo glucémico e instrucción de comunicarse con el médico de cabecera para interpretación de los resultados ( $n=150)$; 3 ) cuidados habituales más autocontrol intensivo con automonitoreo y entrenamiento adicional para interpretación y aplicación de los resultados con el objetivo de acentuar la motivación y mantener la adherencia a un estilo de vida saludable $(n=151)$.

\section{Medición de resultados principales}

El resultado primario fue la medición de HbA1c a los 12 meses y los secundarios, la tension arterial, el peso, los niveles de colesterol total, la razón* entre colesterol total y el colesterol HDL y el índice de masa corporal. El análisis fue por intención de tratar*.

\section{Resultados principales}

Los tres grupos estuvieron fueron equivalentes en sus características basales. A los 12 meses, la diferencia en los niveles de HbA1c no fue estadísticamente significativa $(p=0,12)$ entre los tres grupos, no habiéndose hallado tampoco, diferencias significativas en los diferentes subgrupos de pacientes de acuerdo a la duración de su enfermedad, el tratamiento y las complicaciones. Ver tabla 1.

Tabla 1: evolución de los niveles de hemoglobina glicosilada en cada uno de los grupos con diferente intensidad de automonitorio de la glucemia.

\begin{tabular}{|c|c|c|c|c|}
\hline & \multirow{2}{*}{$\begin{array}{l}\text { Control } \\
(n=152)\end{array}$} & \multicolumn{2}{|c|}{ Intensidad del auto-monitoreo } \\
\hline & & & Baja $(n=150)$ & Alta $(n=151)$ \\
\hline \multirow{3}{*}{$\begin{array}{l}\text { Valor de } \\
\text { hemoglobina } \\
\text { glicosilada (mg\%) }\end{array}$} & Basal & 7,49 & 7,41 & 7,53 \\
\hline & Al año & 7,49 & 7,28 & 7,36 \\
\hline & Cambio & 0,00 & $-0,14$ (IC95\%: $-0,35$ a 0,07$)$ & $-0,17(-0,37 \%$ a 0,03$)$ \\
\hline \multirow{2}{*}{$\begin{array}{l}\text { Pacientes con al } \\
\text { menos un episodic } \\
\text { de hipoglucemia }\end{array}$} & Grado 2 & 14 & 33 & 43 \\
\hline & Grado 3 & 1 & 0 & 0 \\
\hline
\end{tabular}

Grados de la hipoglucemia: grado 1 es la que resuelve sola; en el grado 2 se necesita ayuda; y la de grado 3 cursa con inconciencia o convulsión.

\section{Conclusiones}

En pacientes con diabetes tipo 2 no insulino requirientes, el automonitoreo de la glucemia no se asoció a una mejoría significativa del control glucémico a los 12 meses de tratamiento.

Palabras claves: diabetes tipo 2, automonitoreo, glucemia, $\mathrm{HbA} 1 \mathrm{c}$, grupos paralelos. Key words: type 2 diabetes, self-monitoring, glycemia, HbA1c, parallel groups. Fuente de financiamiento: Servicio Nacional de Salud, Instituto Nacional de Salud e Instituto Nacional para la Investigación en Salud del del Reino Unido de Gran Bretaña. Conflictos de interés: no referidos. Aprobado por el Comité de Etica de Investigaciones de Oxfordshire

\section{Comentario}

Si bien para las Guías de 2000 de la Asociación Latinoamericana de Diabetes "el automonitoreo en sangre capilar utilizando tirillas reactivas y un glucómetro para su lectura es el método ideal" para el control del diabético de tipo 2, ya éstas advertían que "su costo y necesidad de educación y entrenamiento pueden volverlo difícil de aplicar en algunos lugares". La Guía Global 2005 de la Federación Internacional de Diabetes definió la evidencia que lo sustenta como "bastante poco satisfactoria ${ }^{1,2}$ ya que los estudios sobre automonitoreo en los pacientes con diabetes tipo 2 eran de corta duración, con pocos individuos, con inconsistencias en el monitoreo y en el entrenamiento de pacientes, y sin estratificación por el tipo de tratamiento. Un metanálisis realizado en 2000 encontró ocho ensayos clínicos aleatorizados pero ninguna evidencia de la efectividad clínica de este componente de la atención diabetológica ${ }^{3}$. Estos criterios no incluyen a los diabéticos que requieren insulina, en quienes existe consenso en que el automonitoreo es una herramienta importante para detectar variabilidad en los valores de glucemia y corregirla.

\section{Conclusiones del comentador}

La intensidad del automonitoreo de la glucemia no se asociaría a mayor efectividad clínica en diabéticos tipo 2 que no requieren insulina. El tema ya ha generado investigaciones sobre la conducta de los pacientes ${ }^{4}$. La incorporación de nuevos trabajos con mayor poder estadístico podría conducir a rever la indicación rutinaria del automonitoreo. La legislación Argentina vigente (Nacional y Provincial) constituiría un obstáculo para un cambio racional de conducta, ya que ha sido desarrollada pensando en subgrupos de enfermos sin tener en cuenta la salud de la población como una cuestión global.

Ricardo Sarandría [ Médico de Familia. Policlínico Modelo de Cipolletti. ricardo.sarandria@gmail.com ]

Ver glosario*

Sarandría R. El automonitoreo no mejoraría el control del los diabéticos tipo 2 no insulino requirientes. Evid. actual. práct. ambul; 10(5): 137, SepOct.2007. Comentado de: Farmer A, Wade A, Gonder E y col, para el Diabetes Glycaemic Education and Monitoring Trial Group Impact of self monitoring of blood glucose in the management of patients with non-insulin treated diabetes: open parallel group randomised trial. BMJ 2007; 335: 132-6. PMID: 17591623. Disponible en URL: http://www.bmj.com/cgi/reprint/335/7611/132

\section{Referencia}

1. McIntosh A, Hutchinson A, Home PD et al. Clinical guidelines and evidence review for Type 2 diabetes: management of blood glucosa. Sheffield: ScHARR, University Scheffield, 2001. Disponible en URL: www.nice.org.uk/pdf/NICE_full_blood_glucose.pdf

2. Canadian Diabetes Association Clinical Practice Guidelines Expert Committee. Canadian Diabetes Association 2003 Clinical Practice Guidelines for the Prevention and Management of Diabetes in Canada. Canadian Journal of Diabetes 2003; 27(Suppl 2): S18-S23. Disponible en URL: www.diabetes.ca

3. Coster S, Gulliford MC, Seed PT. Self-monitoring in Type 2 diabetes: a meta-analysis.

Diabet Med 2000; 17: 755-61.

4. Peel E., Douglas M., Lawton J Self monitoring of blood glucose in type 2 diabetes: longitudinal qualitative study of patients' perspectives. BMJ 2007;335:493 (8 September), doi:10.1136/bmj.39302.444572.DE 